\title{
Global burden, distribution, and interventions for infectious diseases of poverty
}

\author{
Zulfigar A Bhutta ${ }^{1,2^{*}}$, Johannes Sommerfeld ${ }^{3}$, Zohra S Lassi ${ }^{4}$, Rehana A Salam ${ }^{4}$ and Jai K Das ${ }^{4}$
}

\begin{abstract}
Infectious diseases of poverty (IDoP) disproportionately affect the poorest population in the world and contribute to a cycle of poverty as a result of decreased productivity ensuing from long-term illness, disability, and social stigma. In 2010, the global deaths from HIV/AIDS have increased to 1.5 million and malaria mortality rose to 1.17 million. Mortality from neglected tropical diseases rose to 152,000, while tuberculosis killed 1.2 million people that same year. Substantial regional variations exist in the distribution of these diseases as they are primarily concentrated in rural areas of Sub-Saharan Africa, Asia, and Latin America, with geographic overlap and high levels of co-infection. Evidence-based interventions exist to prevent and control these diseases, however, the coverage still remains low with an emerging challenge of antimicrobial resistance. Therefore, community-based delivery platforms are increasingly being advocated to ensure sustainability and combat co-infections.

Because of the high morbidity and mortality burden of these diseases, especially in resource-poor settings, it is imperative to conduct a systematic review to identify strategies to prevent and control these diseases. Therefore, we attempted to evaluate the effectiveness of one of these strategies, that is community-based delivery for the prevention and treatment of IDoP. In this paper, we describe the burden, epidemiology, and potential interventions for IDoP. In subsequent papers of this series, we describe the analytical framework and the methodology used to guide the systematic reviews, and report the findings and interpretations of our analyses of the impact of community-based strategies on individual IDoPs.
\end{abstract}

Keywords: Infectious diseases of poverty, Neglected tropical diseases malaria, HIV/AIDS, Tuberculosis, Community-based interventions, Community platforms, Community-health workers

\section{Multilingual abstracts}

Please see Additional file 1 for translations of the abstract into the six official working languages of the United Nations.

\section{Introduction}

The Global Burden of Disease Study 2010 reports an increase of 111,000 deaths globally attributable to malaria and neglected tropical diseases (NTDs) (including chagas, leishmaniasis, African trypanosomiasis, schistosomiasis, cysticercosis, echinococcosis, dengue, rabies, ascariasis, as well as other NTDs) in the last two

\footnotetext{
* Correspondence: zulfiqar.bhutta@aku.edu

${ }^{1}$ Center of Excellence in Women \& Child Health, The Aga Khan University, Karachi, Pakistan

${ }^{2}$ Center for Global Child Health Hospital for Sick Children, Toronto, Canada

Full list of author information is available at the end of the article
}

decades, with substantial regional variations and SubSaharan Africa accountable for most of the premature mortalities $[1,2]$. In 2010, the global deaths from human immunodeficiency virus (HIV)/ acquired immunodeficiency syndrome (AIDS) have increased to 1.5 million, and malaria mortality rose to 1.17 million. Mortality from NTDs rose to 152,000 , while tuberculosis (TB) killed 1.2 million people that same year [2]. The United Nations (UN) Millennium Declaration which translated into the Millennium Development Goals (MDGs) underscored the need to combat HIV/AIDS, malaria, and $\mathrm{TB}$, while other infectious diseases including most of the tropical diseases collectively termed as NTDs have slipped into a 'neglected' category, by default [3-6]. NTDs along with HIV, TB, and malaria are collectively referred to as 'infectious diseases of poverty' (IDoPs), and these are primarily concentrated in rural areas of
( Biomed Central

(c) 2014 Bhutta et al.; licensee BioMed Central Ltd. This is an Open Access article distributed under the terms of the Creative Commons Attribution License (http://creativecommons.org/licenses/by/2.0), which permits unrestricted use, distribution, and reproduction in any medium, provided the original work is properly credited. The Creative Commons Public Domain Dedication waiver (http://creativecommons.org/publicdomain/zero/1.0/) applies to the data made available in this article unless otherwise stated. 
Sub-Saharan Africa, Asia, and Latin America, with geographic overlap resulting in high levels of co-infection [7-11]. It is estimated that more than $90 \%$ of the total impact as a result of death and disability caused by neglected diseases occurs in Sub-Saharan Africa [12].

IDoP disproportionately affect the poorest populations in the world and contribute to a cycle of poverty as a result of decreased productivity ensuing from long-term illness, disability, and social stigma [3,5]. The affected populations usually have fewer material, physical, and financial resources to draw from and limited or no access to integrated health care, prevention tools and medications, thus resulting in the most severe adverse impacts. Various social determinants also compound the issue and these include gender dilemmas, unemployment, illiteracy, poor nutrition, indoor air pollution, political instability, and lack of access to proper sanitation and health education, among others [3]. The socioeconomic and physical conditions of those living in poverty create environments that facilitate the transmission of vectors and pathogens consequently leading to long-term illness that further exacerbates poverty by diminishing productivity. Furthermore, global attention and resources have been focused on HIV/AIDS, malaria, and TB as these are specifically outlined in MDG 6, while NTDs have consequently been relegated into the group of "other diseases", and until 2010 , NTDs represented only $0.6 \%$ of the total international development assistance for health [13] despite affecting as many poor people as the big three diseases.

A large proportion of these infectious diseases in lowmiddle- income countries (LMICs) are entirely avoidable or treatable with existing medicines or interventions [14]. Effective and simple interventions to prevent and treat these infectious diseases exist but their delivery to affected populations has proven very difficult due to weak health system infrastructure in many developing countries, thus the need to shift the focus from institutional care delivery to community platforms for improved accessibility. This paper aims to review the disease burden, distribution, existing interventions, and coverage for the prevention and control of IDoP (including NTDs, malaria, TB, and HIV/AIDS), and is followed by a series of papers evaluating the effectiveness of community delivered interventions for the prevention and control of each IDoP.

\section{Review: disease distribution, burden, and consequences}

NTD are a group of 17 bacterial, parasitic protozoal, and viral infections (including dengue, rabies, chagas disease, human African trypanosomiasis, leishmaniasis, cysticercosis/taeniasis, dracunculiasis, echinococcosis, foodborne trematodiases, treponematoses, lymphatic filariasis, onchocerciasis, schistosomiasis, soil-transmitted helminthiases, buruli ulcer, leprosy, trachoma, and yaws) that are chronic and particularly endemic amongst the population in tropical and subtropical regions (Figure 1) [15]. The most common NTDs are a group of helminthic infections affecting one-third of the almost three billion people living on less than USD \$2 per day in developing regions of Sub-Saharan Africa, Asia, and the Americas $[8,16]$.

In 2012, malaria was responsible for over 1.1 million deaths globally [2] and was endemic in 104 countries with substantial geographic disparities. Around $81 \%$ of the malaria incidence and $91 \%$ of the malaria deaths in 2010 occurred in Africa and 86\% involved children under five years of age [17]. A number of factors account for the malaria burden in developing countries, which include climate change, infrastructure, emerging drug and insecticide resistance, massive population and demographic shifts, and costs of containment and therapy. Malaria transmission also greatly depends on climatic conditions such as rainfall

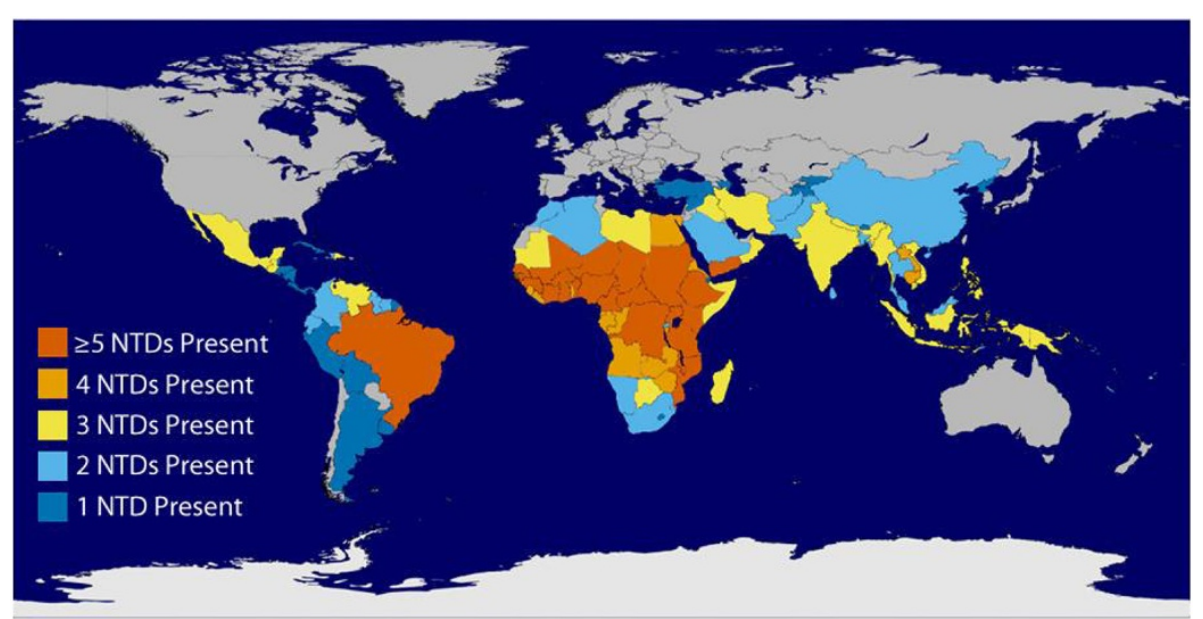

Figure 1 Global overlap of the six most common NTDs. 
patterns, temperature, and humidity. In many endemic areas, transmission is seasonal, with peaks during and just after the rainy season. However, the past decade has seen tremendous expansion in malaria financing and consequent coverage of interventions. Approximately half of countries with ongoing malaria transmission are on track to meet the World Health Assembly (WHA) and Roll Back Malaria (RBM) targets to achieve a $75 \%$ reduction in malaria cases by 2015 , as compared to those in 2000 [18].

More than 65 million people have been infected with HIV and 30 million people have died due to AIDSrelated causes since the emergence of AIDS in 1981 [19]. In 2010, HIV was responsible for approximately 1.5 million deaths [2]. It has an extremely uneven geographical distribution, with Sub-Saharan Africa bearing more than two-thirds of the global burden [20], followed by Asia and the Pacific, where nearly 372,000 people became newly infected in 2011 [21]. Of the 34 million people living with HIV as of 2011, 3.3 million were children under 15 years and 16.7 million were women [21]. Adolescents are also vulnerable as an estimated 2.1 million adolescents (aged 10-19 years) were living with HIV in 2012 in LMICs, with the prevalence among young women twice as high as that among young men throughout Sub-Saharan Africa [22]. Progress has been made on some fronts. The UNAIDS World AIDS Day Report 2012 reported a 50\% reduction in HIV incidence in 25 LMICs between 2001 and 2011 [21], while in SubSaharan Africa, the number of newly infected children declined by $24 \%$ between 2009 and 2011 [20]. With this burden of HIV, the susceptibility of co-infection with leishmaniasis and $\mathrm{TB}$ also rises, and despite proper treatment, relapse is common and often results in death [23].

TB is the second greatest killer worldwide due to a single infectious agent after HIV/AIDS. In 2010, 1.2 million deaths were attributable to TB [2]. Over $95 \%$ of TB deaths occur in LMICs with the highest burden in Asia and Africa. The African region has $24 \%$ of the world's $\mathrm{TB}$ cases and the highest rates of cases and deaths per capita, while India and China together account for almost $40 \%$ of the world's TB burden [24]. There is also the emerging issue of multi-drug resistant TB (MDR$\mathrm{TB})$, which is rising and reached 60,000 in the 27 high MDR-TB burden countries worldwide in 2011 [24]. Although the MDG target to halt and reverse the TB epidemic by 2015 is already achieved, the disease burden remains enormous with resurgence in many areas due to HIV/AIDS. An estimated $13 \%$ of the TB cases in 2011 were co-infected with HIV and 430,000 deaths were among the HIV-positive population [24].

As a group, IDoP are amongst the top ten causes of disability-adjusted years (DALYs) (see Table 1). They can lead to burdensome health consequences that include
Table 1 Estimated number of disability-adjusted life years (DALYs) (in thousands) by IDoP

\begin{tabular}{ll}
\hline Infectious diseases of poverty & $\begin{array}{l}\text { Estimated DALYs } \\
\text { (in thousands) }\end{array}$ \\
\hline Neglected tropical diseases & 1,243 \\
Viral: Dengue & 2,297 \\
Rabies & 1,346 \\
Protozoan: Human African trypanosomiasis & 499 \\
Chagas disease & 3,754 \\
Leishmaniasis & 503 \\
Helminth: Cysticercosis/Taeniasis & - \\
Dracunculiasis & 600 \\
Echinococcosis & 665 \\
Food borne trematodiasis & 2,740 \\
Lymphatic filariasis & 564 \\
Onchocerciasis & 3,971 \\
Schistosomiasis & 1,254 \\
Ascariasis & 630 \\
Trichuriasis & 3,159 \\
Hookworm disease & - \\
Bacterial: Buruli ulcer & 215 \\
Leprosy & 308 \\
Trachoma & - \\
Yaws & 42,240 \\
Tuberculosis & 95,226 \\
HIV/AIDS & 55,414 \\
Malaria & \\
\hline
\end{tabular}

Source: WHO. Disease Burden: DALY Estimates 2011 and Global Burden of Diseases 2010

blindness due to onchocerciasis and trachoma, and disfigurement from Lymphatic filariasis (LF), leishmaniasis, leprosy and buruli ulcer, leading to severe economic costs [25]. Schistosomiasis can result in severe organ pathology, anemia, malnutrition, and can also increase the risk of HIV. Repeated infection with trachoma can lead to scarring of the inside of the eyelid such that it turns inward, resulting in trichiasis and scar of the cornea. If untreated, irreversible corneal opacities are formed and blindness ensues. Ascariasis, trichuriasis, schistosomiasis, hookworms, malaria, and TB can lead to malnutrition and anemia, which is of particular concern for young children and pregnant women who are especially prone to the adverse health consequences of undernutrition. Most of these infections affect children and young adults leading to the loss of their most productive years, and some of these diseases can be fatal if left untreated [6]. Infections of severe intensity can impair physical growth and cognitive development and are a cause of micronutrient deficiencies leading to poor school performance and absenteeism in children, reduced work productivity in 
adults, and adverse pregnancy outcomes [26]. Malaria during pregnancy may lead to severe disease, spontaneous abortions, preterm birth, low birth weight, and anemia.

In addition to the grave health consequences, this group of diseases also leads to huge economic costs for both the individual and society. Leishmaniasis results in approximately USD \$1.3 billion/years lost in productivity, while for trachoma the loss in productivity is estimated at USD $\$ 2.9$ billion [27]. In India, the average total economic burden for dengue fever is approximately USD \$29.3 million, and schistosomiasis in the Philippines results in 45.4 days off-work lost per infected person/year [23]. Direct costs associated with malaria-encompassing illnesses, treatment, and premature deaths have been estimated to be at least USD $\$ 12$ billion per year, however, the costs are often more than that in terms of lost economic growth [27]. Conversely, controlling these diseases has the potential to increase productivity as deworming against soil-transmitted helminthiasis ( $\mathrm{STH})$ in Kenya has shown to increase the present wages by more than USD $\$ 40$ per treated person, with a benefit-to-cost ratio of 100 [28].

\section{Interventions and coverage}

The World Health Organization (WHO) promotes the use of five public health strategies to control, eliminate, and eradicate NTDs. These include preventive chemotherapy; innovative and intensified disease-management; vector control and pesticide management; provision of safe drinking water, basic sanitation and hygiene, and education; and veterinary public health services [29]. Mass drug administration (MDA) has been a major approach to combat helminthiasis including ascariasis, trichuriasis, hookworm, schistosomiasis, LF, onchocerciasis, and trachoma in developing countries [30]. There is evidence suggesting significant benefits of treating diagnosed cases [31,32], and these medicines are not only effective but also cost efficient. A USD $\$ 0.50$ package containing several inexpensive and safe drugs can treat more than 15 types of neglected parasitic infections [33].

The WHO recommends periodic preventive treatment with anthelmintics for all at-risk people living in endemic areas to reduce morbidity by reducing the worm burden. Large-scale and successful control activities implemented during 2001-2010 demonstrate the feasibility of mass deworming, and these experiences have informed the development of tools to facilitate its implementation [34]. Of the 123 countries requiring preventive chemotherapy, 40 require interventions for three or more diseases and 33 of these 40 countries are in Africa [23,29]. About 1.9 billion people require preventive chemotherapy for at least one NTD; $55 \%$ of them require preventive treatment for one or two diseases, and $45 \%$ require it for three or more diseases $[23,29]$. However, by the end of 2010 , only 25 countries had achieved at least one of the targets set for delivering preventive chemotherapy for LF, onchocerciasis or STH, and only five of these countries had reached the targets for delivering preventive chemotherapy for three or more diseases simultaneously $[23,29]$. Therefore, interventions need to be scaled-up considerably if targets set in the WHA's resolutions are to be met [29].

Key interventions recommended by the WHO to prevent and control malaria include prompt and effective treatment with artemisinin-based combination therapies; the use of insecticide-treated nets (ITNs); and indoor residual spraying (IRS) with insecticide to control vector mosquitoes. In the past decade, the percentage of households owning at least one ITN in Sub-Saharan Africa reached an estimated $53 \%$ by 2011 and remained at 53\% in 2012. It must be noted however that this is greatly challenged by the limited deliveries of ITNs and increasing mosquito resistance to insecticides [18]. In 2011, the proportion of pregnant women attending an antenatal care clinic and receiving two doses of intermittent preventive treatment during pregnancy (IPTp) ranged from $30 \%$ to $57 \%$ in 2011 , however, intermittent preventive treatment for infants and seasonal malaria chemoprevention for children still await to be adopted by endemic countries $[18,35]$. An emerging challenge arising due to over diagnosis and treatment is the drug resistance to artemisinins detected in four countries of the Greater Mekong Subregion: Cambodia, Myanmar, Thailand, and Vietnam [18]. More recently, the WHO has introduced a new initiative, entitled "T3: Test, Treat and Track". It urges malaria-endemic countries and donors to move towards universal access to diagnostic testing and antimalarial treatment to build robust control and surveillance systems. The current recommendation of diagnostic confirmation of malaria before treatment initiation was adopted by 41 countries in the African region, however, this practice attained less than 50\% coverage in 2011 even in public health facilities [35].

Strategies for HIV prevention involve risk-reduction through education and counseling. The WHO has recommended key approaches which include condom use, testing and counseling, male circumcision, preventive antiretroviral therapy (ART), harm reduction for injecting drug users, and elimination of mother-to-child transmission (MTCT) of HIV [36,37]. In 2012, 9.7 million people in LMICs received ART (representing $61 \%$ of all who were eligible), however, under the 2013 WHO guidelines, this treatment coverage represents only $34 \%$ of the 28.3 million people eligible [22]. Although the coverage of effective ART regimens in LMICs for preventing MTCT was 57\% in 2011, a lot is still desired to eliminate it completely as a recent report suggested that, on average, nearly half of all children newly infected with HIV in the 20 African countries surveyed were acquiring HIV during breastfeeding because of 
low ART coverage during this period. In 2012, 375,000 more pregnant women living with HIV received ART medicines than in 2009 [38].

TB is preventable as well as curable, and its transmission could be prevented by prompt identification and treatment of the infection. The WHO is working to dramatically reduce the burden of TB and halve TB deaths and prevalence by 2015 through its Stop TB Strategy and by supporting the Global Plan to Stop TB. Between 1995 and 2011, 51 million people were successfully treated for TB in countries that had adopted the WHO strategy, saving 20 million lives [24]. There has also been progress in implementing collaborative $\mathrm{TB} / \mathrm{HIV}$ activities as recommended by the WHO in 2004 [24].

\section{The changing care paradigm}

A large proportion of infectious diseases in LMICs are entirely avoidable or treatable with existing medicines or interventions which are also highly cost effective, however, their delivery to the affected populations has proven very difficult due to weak health systems and infrastructures [14]. Another major issue is access to and utilization of health services, which has been a concern in LMICs, with not enough progress being made on various health parameters. Other impeding factors include gender discrimination, low levels of female literacy, and lack of women empowerment; all of these prevent women from seeking care for themselves and their children. Health care is also unaffordable for many families due to economic barriers including formal and informal healthcare fees, cost of medicines and tests, cost of not working during hospitalization, travel, food, and accommodation.

Effective delivery of proven interventions require a variety of components ranging from training health workers, effective use of epidemiological data, proper delivery of safe medicines and commodities, accurate monitoring and evaluation, and providing feedback to the community. Successful implementation requires a positive interrelation between programs for disease control and the health system at large. Global health initiatives have created a complex health system with an increasing number of actors entering the field and implementing diverse health systems strategies [39]. These health system issues need to be acknowledged and worked on [40]. Therefore, a combination of public health strategies is required to achieve control of each of these diseases and an integrated approach to disease control and elimination is strongly advised especially in high-burden countries [29]. There are also wide inequities in access to and utilization of healthcare amongst the wealth quintiles. Poor children are more likely to be exposed to health risks, and they have less resistance to diseases because of undernutrition and other hazards. These inequities are compounded by reduced access to preventive and curative interventions [41].
Community-based interventions (CBIs) have the potential to overcome the barriers of access and availability and, if adequately equipped and supported by parallel structures, can make a significant impact on reducing the burden of IDoP [42-44]. However, a major issue is the availability of a trained health force to scale-up these interventions in population settings. According to a 2006 report by the WHO, 57 countries from Africa and Asia are facing shortages of a healthcare workforce and a total estimate of 4,250,000 workers are needed to fill the gap [45]. For scaling-up these interventions, there is a need to strengthen health systems [46], and also to develop alternative cadres for task shifting from trained healthcare workers to lay workers and from specialized facilities to community-based delivery [47]. Many of the interventions targeting infectious diseases have been administered via community platforms through the community health workers (CHWs) who have received basic training [48-50]. Although CHWs may not be able to replace the need for sophisticated healthcare delivery, they can play an important role in providing access to health care and services for the unreachable. Successful examples exist, for example, in Brazil, where CHWs provide coverage to over 60 million people [51]. Ethiopia is training about 30,000 workers with emphasis on maternal and child health, HIV, and malaria. Other similar programs are also being considered in countries such as India, Ghana, and South Africa. Apart from providing chemotherapy, CHWs can also play a major role in imparting health education regarding general hygiene and sanitation and intervene for vector control measures within household and community settings. These community delivery strategies are not only effective but are also cost efficient, and by training teachers and other school personnel to administer anthelmintic drugs, costs could be reduced by "piggy-backing" on existing programs in the educational sector [52]. In Ghana and Tanzania, delivery of school-based targeted anthelmintic treatment cost as little as US $\$ 0.03$ per child, which is as low as one-tenth of the estimated costs for vertical delivery [52].

\section{Conclusion}

In order to evaluate the effectiveness of CBIs, we developed an analytical framework and conducted systematic reviews of the existing studies focusing on CBIs for prevention and control of helminthic and non-helminthic NTDs, malaria, TB, and HIV/AIDS compared to the routine healthcare delivery. For this review, we categorized NTDs into helminthic and non-helminthic diseases, and reported the findings accordingly in separate papers. Helminthic diseases included soil-transmitted helminthiasis (ascariasis, hookworm, and trichuriasis) along with schistosomiasis, lymphatic filariasis, onchocerciasis, and 
dracunculiasis. Non-helminthic diseases included dengue, African trypanosomiasis, chagas, leishmaniasis, trachoma, leprosy, and buruli ulcer. In this series of eight papers, we describe the analytical framework and methodology used for the systematic reviews, and report findings on the effectiveness of CBIs for the prevention and control of helminthic NTDs, non-helminthic NTDs, malaria, HIV/AIDS, and tuberculosis. In the final paper, we propose a way forward.

\section{Additional file}

\section{Additional file 1: Multilingual abstracts in the six official working} languages of the United Nations.

\section{Abbreviations \\ ART: Antiretroviral therapy; CBI: Community-based intervention; CHW: Community health worker; DALY: Disability-adjusted life year; HIV/ AIDS: Human immunodeficiency virus/acquired immunodeficiency syndrome; IDoP: Infectious disease of poverty; IPTp: Intermittent preventive therapy during pregnancy; IRS: Indoor residual spraying; ITN: Insecticide- treated net; LF: Lymphatic filariasis; LMIC: Low- middle- income country; MDA: Mass drug administration; MDG: Millennium development goal; MDR-TB: Multi drug resistant tuberculosis; MTCT: Mother-to-child transmission; NTD: Neglected tropical disease; RBM: Roll back malaria; STH: Soil-transmitted helminthiasis; TB: Tuberculosis; UN: United nations; WHA: World health assembly; WHO: World health organization.}

\section{Competing interests}

The authors declare that they have no financial or non-financial competing interests.

\section{Authors' contributions}

ZAB and JS were responsible for designing and coordinating the review. All authors contributed, read and approved the final manuscript.

\section{Acknowledgements}

The collection of scoping reviews in this special issue of Infectious Diseases of Poverty was commissioned by the UNICEF/UNDP/World Bank/WHO Special Programme for Research and Training in Tropical Diseases (TDR) in the context of a Contribution Agreement with the European Union for

"Promoting research for improved community access to health interventions in Africa".

\section{Author details}

${ }^{1}$ Center of Excellence in Women \& Child Health, The Aga Khan University, Karachi, Pakistan. ${ }^{2}$ Center for Global Child Health Hospital for Sick Children, Toronto, Canada. ${ }^{3}$ UNDP/World Bank/WHO Special Programme for Research and Training in Tropical Diseases (TDR), Geneva, Switzerland. ${ }^{4}$ Division of Women and Child Health, The Aga Khan University, Karachi, Pakistan.

Received: 5 January 2014 Accepted: 18 July 2014

Published: 31 July 2014

\section{References}

1. WHO: Disease Burden: DALY Estimates. 2011. Available at http://www.who.int/ healthinfo/global_burden_disease/gbd/en/.

2. Lozano R, Naghavi M, Foreman K, Lim S, Shibuya K, Aboyans V, Abraham J, Adair T, Aggarwal R, Ahn SY, AlMazroa MA, Alvarado M, Anderson HR, Anderson LM, Andrews KG, Atkinson C, Baddour LM, Barker-Collo S, Bartels DH, Bell ML, Benjamin EJ, Bennett D, Bhalla K, Bikbov B, Abdulhak AB, Birbeck G, Blyth F, Bolliger I, Boufous S, Bucello C: Global and regional mortality from 235 causes of death for 20 age groups in 1990 and 2010: a systematic analysis for the Global Burden of Disease Study 2010. Lancet 2012, 380(9859):2095-2128.
3. Manderson L, Aagaard-Hansen J, Allotey P, Gyapong M, Sommerfeld J: Social research on neglected diseases of poverty: continuing and emerging themes. PLoS Negl Trop Dis 2009, 3(2):0.

4. World Health Organization: Global Report for Research on Infectious Diseases of Poverty. 2012

5. Molyneux DH, Hotez PJ, Fenwick A: "Rapid-Impact Interventions": how a policy of integrated control for Africa's neglected tropical diseases could benefit the poor. PLoS Med 2005, 2(11):e336.

6. Zhang Y: Helen Keller International Position Paper Control of Neglected Tropical Diseases. 2010.

7. Morens DM, Folkers GK, Fauci AS: The challenge of emerging and re-emerging infectious diseases. Nature 2004, 430(6996):242-249.

8. Hotez PJ, Molyneux DH, Fenwick A, Ottesen E, Sachs SE, Sachs JD: Incorporating a rapid-impact package for neglected tropical diseases with programs for HIV/AIDS, tuberculosis, and malaria. PLOS Med 2006 3(5):e102.

9. McKenzie FE: Polyparasitism. Int J Epidemio/ 2005, 34(1):221-222. author reply 222-3.

10. Molyneux DH, Nantulya VM: Linking disease control programmes in rura Africa: a pro-poor strategy to reach Abuja targets and millennium development goals. BMJ 2004, 328(7448):1129-1132.

11. Raso G, Luginbühl A, Adjoua CA, Tian-Bi NT, Silué KD, Matthys B, Vounatsou $P$ Wang Y, Dumas ME, Holmes E, Singer BH, Tanner M, N'goran EK, Utzinger J: Multiple parasite infections and their relationship to self-reported morbidity in a community of rural Cote d'Ivoire. Int J Epidemiol 2004, 33(5):1092-1102.

12. Liu L, Johnson HL, Cousens S, Perin J, Scott S, Lawn JE, Rudan I, Campbell H, Cibulskis R, Li M, Mathers C, Black RE, Child Health Epidemiology Reference Group of WHO and UNICEF: Global, regional, and national causes of child mortality: an updated systematic analysis for 2010 with time trends since 2000. Lancet 2012, 379(9832):2151-2161.

13. Liese $B H$, Schubert $L$ : Official development assistance for health-how neglected are neglected tropical diseases? An analysis of health financing. Int Health 2009, 1(2):141-147.

14. WHO: Integrated Community-based Interventions. TDR Business Line 11. Geneva: World Health Organization; 2009.

15. WHO: Neglected Tropical Diseases. 2014. Available at http://www.who.int/ neglected_diseases/diseases/en/. Access date: May 29, 2014.

16. Hotez PJ, Molyneux DH, Fenwick A, Kumaresan J, Sachs SE, Sachs JD, Savioli L: Control of neglected tropical diseases. N Engl J Med 2007, 357(10):1018-1027.

17. Nsungwa-Sabiiti J, Peterson S, Pariyo G, Ogwal-Okeng J, Petzold MG, Tomson G: Home-based management of fever and malaria treatment practices in Uganda. Trans R Soc Trop Med Hyg 2007, 101:1199-1207.

18. WHO: WHO Global Malaria Programme: World Malaria Report 2011. 2012.

19. World Bank and HIV/AIDS: The Facts; 2012. Fact sheet]. Available from: http:// www.unaids.org/en/resources/presscentre/factsheets/

20. Sub-Saharan Africa, in Regional Fact Sheet 2012, The Joint United Nations Programme on HIV/AIDS (UNAIDS). 2012, Available at http:// www.unaids.org/en/media/unaids/contentassets/documents/ epidemiology/2012/gr2012/2012_FS_regional_ssa_en.pdf.

21. UNAIDS. World AIDS Day report: Results, 2012, Joint United Nations Programme on HIV/AIDS (UNAIDS): Geneva, Switzerland. 2012, Available at http://www.unaids.org/en/resources/publications/2012/name,76120,en.asp.

22. UNAIDS: Global Report 2012: UNAIDS Report on the Global AIDS Epidemic2013. Available at http://www.unaids.org/en/resources/campaigns/ globalreport2013/globalreport/.

23. World Health Organization. Dept. of Control of Neglected Tropical, Diseases: Working to overcome the global impact of neglected tropical diseases: first WHO report on neglected tropical diseases. Geneva, Switzerland: World Health Organization; 2010

24. WHO: Global Tuberculosis report 2012; 2012

25. World Health Organization: The global burden of disease: 2004 update. 2008. Geneva: World health organization; 2011.

26. Hall A, Hewitt G, Tuffrey $V$, De Silva N: A review and meta-analysis of the impact of intestinal worms on child growth and nutrition. Matern Child Nutr 2008, 4(Suppl 1):118-236.

27. Conteh L, Engels T, Molyneux DH: Socioeconomic aspects of neglected tropical diseases. Lancet 2010, 375(9710):239-247.

28. Ter Kuile FO, Terlouw DJ, Phillips-Howard PA, Hawley WA, Friedman JF, Kolczak MS, Kariuki SK, Shi YP, Kwena AM, Vulule JM, Nahlen BL: Impact of permethrin-treated bed nets on malaria and all-cause morbidity in 
young children in an area of intense perennial malaria transmission in western Kenya: cross-sectional survey. Am J Trop Med Hyg 2003, 68(4 suppl):100-107.

29. World Health Organization: Sustaining the drive to overcome the global impact of neglected tropical diseases. Geneva, Switzerland: World Health Organization; 2013.

30. Mahmoud A, Zerhouni E: Neglected tropical diseases: moving beyond mass drug treatment to understanding the science. Health Affairs 2009, 28(6):1726-1733.

31. Taylor-Robinson D, Jones A, Garner P: Deworming drugs for treating soil-transmitted intestinal worms in children: effects on growth and school performance. Cochrane Database Syst Rev 2012, 4.

32. Haider BA, Humayun Q, Bhutta ZA: Effect of administration of antihelminthics for soil transmitted helminths during pregnancy. Cochrane Database Syst Rev 2012, 2.

33. WHO: Neglected tropical diseases: preventive chemotherapy and transmission control. 2006, Available at http://whqlibdoc.who.int/ publications/2009/9789241598705_eng.pdf. Access date: May 29, 2014.

34. D'Alessandro U, Olaleye BO, McGuire W, Langerock P, Bennett S, Aikins MK, Thomson MC, Cham MK, Cham BA, Greenwood BM: Mortality and morbidity from malaria in Gambian children after introduction of an impregnated bednet programme. Lancet 1995, 345(8948):479-483.

35. Liu XD, Wang XL, Bai X, Liu XL, Wu XP, Zhao Y, Sun SM, Yu L, Su XZ, Wang ZQ, Wang F, Liu MY: Oral administration with attenuated Salmonella encoding a Trichinella cystatin-like protein elicited host immunity. Exp Parasitol 2014, 141:1-11.

36. Clark LF, Miller KS, Nagy SS, Avery J, Roth DL, Liddon N, Mukherjee S: Adult identity mentoring: reducing sexual risk for African-American seventh grade students. J Adolesc Health 2005, 37(4):337.

37. Chhabra R, Springer C, Leu CS, Ghosh S, Sharma SK, Rapkin B: Adaptation of an alcohol and HIV school-based prevention program for teens. AIDS Behav 2010, 14(Suppl 1):S177-S184.

38. Xia S, Liu J: A belief-based model for characterizing the spread of awareness and its impacts on individuals' vaccination decisions. J $R$ Soc Interface 2014, 11(94):20140013.

39. Harris M, Reza JN: Global Report for Research on Infectious Diseases of Poverty. Geneva, Switzerland: World Health Organization; 2012.

40. Huntington D: Health systems perspectives: infectious diseases of poverty. Infect Dis Poverty 2012, 1(1):3.

41. Victora CG, Wagstaff A, Schellenberg JA, Gwatkin D, Claeson M, Habicht JP: Applying an equity lens to child health and mortality: more of the same is not enough. Lancet 2003, 362(9379):233-241.

42. Ansa GA, Walley JD, Siddiqi K, Wei X: Assessing the impact of TB/HIV services integration on TB treatment outcomes and their relevance in TB/HIV monitoring in Ghana. Infect Dis Poverty 2012, 1:13.

43. Dabo A, Bary B, Kouriba B, Sankaré O, Doumbo O: Factors associated with coverage of praziquantel for schistosomiasis control in the community-direct intervention (CDI) approach in Mali (West Africa). Infect Dis Poverty 2013, 2(1):11.

44. Ajayi IO, Jegede AS, Falade CO, Sommerfeld J: Assessing resources for implementing a community directed intervention (CDI) strategy in delivering multiple health interventions in urban poor communities in Southwestern Nigeria: a qualitative study. Infect Dis Poverty 2013, 2(1):25.

45. Fraser B: Human resources for health in the americas. Lancet 2007, 369(9557):179-180

46. Travis P, Bennett S, Haines A, Pang T, Bhutta Z, Hyder AA, Pielemeier NR, Mills A, Evans T: Overcoming health-systems constraints to achieve the Millennium Development Goals. Lancet 2004, 364(9437):900-906.

47. Brady MA, Hooper PJ, Ottesen EA: Projected benefits from integrating NTD programs in sub-Saharan Africa. Trends Parasitol 2006, 22(7):285-291.

48. Lewin S, Munabi-Babigumira S, Glenton C, Daniels K, Bosch-Capblanch X, van Wyk BE, Odgaard-Jensen J, Johansen M, Aja GN, Zwarenstein M, Scheel IB: Lay health workers in primary and community health care for maternal and child health and the management of infectious diseases. Cochrane Database Syst Rev 2010, (Issue 3): Art. No.: CD004015. doi:10.1002/14651858. CD004015

49. Global Health Workforce Alliance, World Health Organization: Global Experience of Community Health Workers for Delivery of Health Related Millennium Development Goals: A Systematic Review, Country Case Studies, and Recommendations for Integration into National Health Systems. Geneva: World Health Organization; 2010.
50. Volmink J, Garner P: Directly observed therapy for treating tuberculosis. Cochrane Database Syst Rev 2007, (4):CD003343

51. World Health Organization: Community Health Workers: what do we know about them? the state of evidence on programmes, activities, costs and impact of health outcomes of using health workers. Geneva: World Health Organization; 2007.

52. Hawley WA, Phillips-Howard PA, ter Kuile FO, Terlouw DJ, Vulule JM, Ombok M, Nahlen BL, Gimnig JE, Kariuki SK, Kolczak MS, Hightower AW: Community-wide effects of permethrin-treated bed nets on child mortality and malaria morbidity in western Kenya. Am J Trop Med Hyg 2003, 68(4):121-127.

doi:10.1186/2049-9957-3-21

Cite this article as: Bhutta et al:: Global burden, distribution, and interventions for infectious diseases of poverty. Infectious Diseases of Poverty 2014 3:21.

\section{Submit your next manuscript to BioMed Central and take full advantage of:}

- Convenient online submission

- Thorough peer review

- No space constraints or color figure charges

- Immediate publication on acceptance

- Inclusion in PubMed, CAS, Scopus and Google Scholar

- Research which is freely available for redistribution

Submit your manuscript at www.biomedcentral.com/submit
C) BioMed Central 